\title{
Obesity prevention through primary health care: Promoting physical activity in early childhood
}

\author{
Alana Maltby
}

\section{University of Western Ontario}

The number of preschool-aged children who are overweight or obese is alarming. In fact, approximately 43 million children worldwide, under the age of five, were considered overweight in $2010 .{ }^{1}$ With obesity rates among children reaching epidemic proportions, increased risk factors for insulin resistance and cardiovascular disease are occurring even earlier in life. ${ }^{1}$ To minimize the effects of obesity and related comorbidities on the health of the population, our focus needs to be directed to early primary prevention. The following commentary will highlight the need for both the encouragement and prescription of physical activity (PA) by primary health care providers to prevent childhood obesity.

\section{The Necessity of Intervening in the Early Years}

Intervening at a young age is critical because lifestyle behaviours (i.e., being physically active) are formed early ${ }^{2}$ and may help prevent chronic disease and obesity from continuing into adulthood. ${ }^{1}$ Furthermore, as preschoolers' behaviours are largely under the control of parents, ${ }^{3}$ involving and educating parents on PA is essential. Childhood obesity has numerous physiological and psychological health implications, ${ }^{4-6}$ and once established, obesity is also very challenging to reduce. ${ }^{1}$ Fortunately, in several controlled interventions involving children, PA has been found to improve body composition and is a promising method of obesity prevention. ${ }^{2}$

\section{Physical Activity Promotion in Early Childhood}

The advantages of PA are numerous (e.g., body weight, improved body composition, psychosocial well-being, etc.), ${ }^{2}$ and yet sedentary behaviours and physical inactivity are becoming increasingly prevalent among young Canadian children. ${ }^{5}$ As activity rates begin to decline as children get older, ${ }^{5}$ it is necessary to promote age-appropriate activities that are enjoyable for preschoolers. The importance of unstructured play (i.e., running, climbing, imaginary play, etc.) during this age is often underscored as a substantial proportion of preschoolers' daily PA. ${ }^{7}$ Encouraging health care providers to emphasize the variance in children's activity preferences and forms of play can assist parents to minimize sedentary behaviour and increase PA. ${ }^{4}$ When prescribing PA as a preventative measure for both children and their families, physicians should promote PA as a healthy lifestyle behaviour rather than a method of weight reduction. ${ }^{3}$ The acknowledgment of PA in this context will frame the discussion in a manner that is nonjudgmental and that does not place blame on either the parent or the child. ${ }^{7,8}$

Pediatricians and family physicians have a unique advantage in the promotion of preventative strategies, as the majority of children this age are seen for routine pediatric care. ${ }^{3,6,8}$ Physicians are advised to assess children's body mass index (over 2 years of age) ${ }^{4}$ and early lifestyle determinants (e.g., PA levels) at routine visits to screen for increased risk of obesity. ${ }^{6}$ Integrating this assessment into practice will help physicians provide anticipatory guidance to parents and families. ${ }^{7}$ Unfortunately, physicians are often met with barriers in obesity prevention among children (e.g., limited hours to provide lifestyle guidance or lack of knowledge in physical activity). ${ }^{4}$ Primary care providers need resources and support for the surveillance of obesity predictors in early childhood. ${ }^{4,6}$ However, inconsistencies remain as to which are the best approaches for this type of assessment, thus highlighting the need for evidence-based preventative strategies. ${ }^{6}$

Acknowledging this research gap, a Canadian primary care practice based research network (PCBRN) called TARGet Kids! is aiming to gather data that will inform evidence-based preventative interventions. ${ }^{6}$ The PCBRN provides an outlet for knowledge translation with primary care providers, researchers, and policymakers. ${ }^{6}$ In comparison, international efforts such as the World Health Organization's 
(WHO) Global Strategy on Diet, Physical Activity and Health aim to establish a framework that examines how behaviour change is impacted by supportive environments, policies, and programs. ${ }^{1}$ Furthermore, WHO also acknowledges that effective obesity prevention efforts must have input from multiple stakeholders. ${ }^{1}$

\section{Strengthening Prevention Through Primary Health Care}

Primary health care embodies various aspects of primary care (i.e., episodic or comprehensive care), public health, and health policy. ${ }^{9}$ Additionally, it is important to recognize that primary health care extends beyond the health care system and encourages intersectoral collaboration and community involvement. ${ }^{10}$ To effectively address the health determinants influencing childhood obesity, collaborative efforts between primary care providers, various sectors (e.g., schools), and governments are crucial. ${ }^{4,6,9}$ Furthermore, improvements in the translation of research are required in order for primary care providers to translate these joint efforts into practice, thereby enhancing prevention methods.

\section{Conclusion}

The use of primary health care as a method for childhood obesity prevention is not currently being utilized to its full potential. ${ }^{6}$ Promoting increased PA appears to be a viable method of obesity prevention, given that lifestyle behaviours are more amenable to change at a young age..$^{5}$ Therefore, establishing collaborative partnerships in primary health care will foster the development of efficacious prevention and surveillance strategies targeting the rising rates of obesity among children.

\section{References}

1. World Health Organization. Population-based prevention strategies for childhood obesity: Report of the WHO forum and technical meeting. Geneva, Switzerland: World Health Organization; 2010.

2. Goran $\mathrm{MI}$, Reynolds $\mathrm{KD}$, Lindquist $\mathrm{CH}$. Role of physical activity in the prevention of obesity in children. Int J Obes. 1999; 23(Suppl 3):S18-S33.

3. Olstad DL, McCargar L. Prevention of overweight and obesity in children under the age of 6 years. Appl Physiol Nutr Metab. 2009;34:551-70.

4. Lau DCW, Douketis JD, Morrison KM, Hramiak IM, Sharma AM, Ur E. 2006 Canadian clinical practice guidelines on the management and prevention of obesity in adults and children [summary]. Can Med Assoc J. 2007;176/8 Suppl):S1-13.

5. Goldfield GS, Harvey A, Grattan K, Adamo KB. Physical activity promotion in the preschool years: a critical period to intervene. Int J Environ Res Public Health. 2012;9:1326-42.

6. Morinis J, Maguire J, Khovratovich M, McCrindle BW, Parkin PC, Birken CS. Paediatric obesity research in early childhood and the primary care setting: The TARGet Kids! research network. Int J Environ Res Public Health. 2012 Apr;9(4):1343-54.

7. Krebs NF, Baker Jr. RD, Greer FR, Heyman MB, Jaksic T, Lifshitz F, et al. Prevention of pediatric overweight and obesity. Pediatrics. 2003;112(2):424-30.

8. Tucker P, Irwin JD, Sangster Bouck LM, He M, Pollett G. Preventing paediatric obesity; Recommendations from a community-based qualitative investigation. Obes Rev. 2006;7(3):251-60.

9. Martin-Misener R, Valaitis R, Wong ST, Macdonald M, Meagher-Stewart D, Kaczorowski J, et al. A scoping literature review of collaboration between primary care and public health. Prim Health Care Res Dev. 2012;13(4):32746.

10. Walley J, Lawn JE, Tinker A, de Francisco A, Chopra M, Rudan I, et al. Primary health care: Making Alma-Ata a reality. Lancet. 2008;372(9642):1001-7.

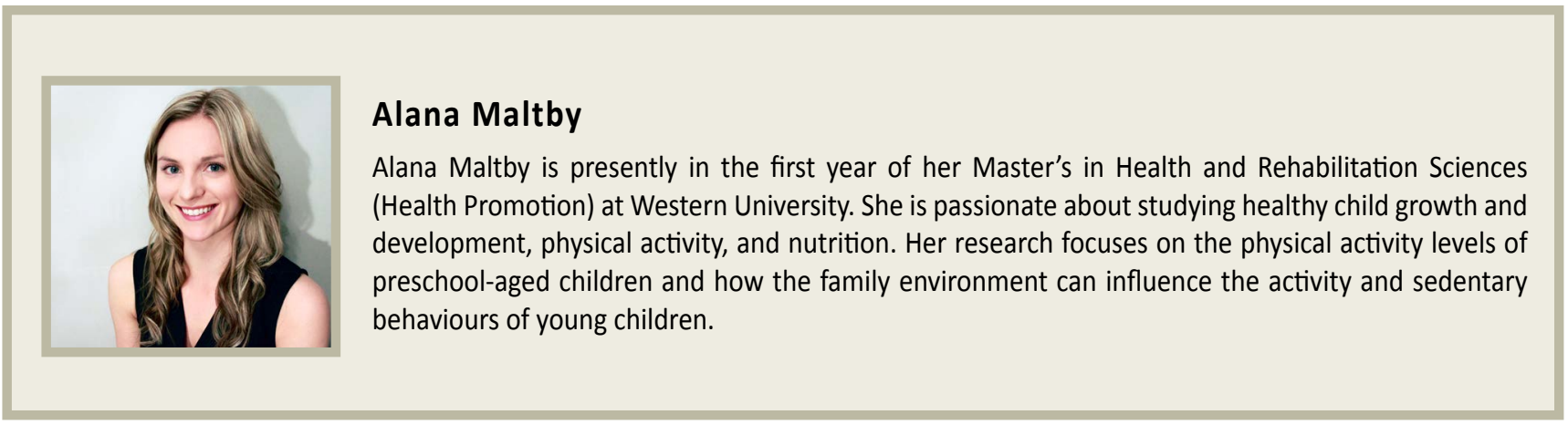

